\title{
A Knowledge Representation Model for Massive Open Online Course Platforms
}

Kiselev, B., Yakutenko, V., Yuriev, M., Zhelbakov, I. and Cunningham, S.

This is a paper presented at the 7th IEEE Int. Conference on Internet Technologies and Applications ITA-17, Wrexham, UK, 12-15 September 2017.

Copyright of the author(s). Reproduced here with their permission and the permission of the conference organisers.

\section{Recommended citation:}

Kiselev, B., Yakutenko, V., Yuriev, M., Zhelbakov, I. and Cunningham, S. (2017) 'A Knowledge Representation Model for Massive Open Online Course Platforms'. In: Proc. 7th IEEE Int. Conference on Internet Technologies and Applications ITA-17, Wrexham, UK, 12-15 September 2017, pp. 169-172. doi: 10.1109/ITECHA.2017.8101931 


\title{
A Knowledge Representation Model for Massive Open Online Course Platforms
}

\author{
Boris Kiselev, Viacheslav Yakutenko, Mikhail Yuriev \\ Department of Informatics and Control Processes \\ National Research Nuclear University "MEPhI" \\ Moscow, Russia
}

\author{
Igor Zhelbakov \\ Institute of Automatics and Computer Engineering \\ National Research University "MPEI" \\ Moscow, Russia
}

\author{
Stuart Cunningham \\ School of Applied Science, Computing and Engineering \\ Wrexham Glyndŵr University \\ Plas Coch, Mold Road, Wrexham, LL11 2AW, UK
}

\begin{abstract}
This paper describes a knowledge model for the design of Massive Open Online Course (MOOC) platforms. It is based on our generic instructional engineering method called Knowledge Field of Educational Environment with Competence Boundary Conditions (KFEEC). KFEEC uses the ontology as a foundation for the knowledge representation model. It provides a flexible structure to the various self-paced e-learning system designs but appears to be overcomplicated for the MOOC platform. This paper describes the KFEEC method, the steps of adapting the KFEEC to the MOOC platform design, and the specification of the resulting knowledge model. This model is a core of the MOOC platform that will be developed in future work.
\end{abstract}

Keywords-MOOC; e-learning; ontology; knowledge representation; instructional design; $R D F$

\section{INTRODUCTION}

Massive Open Online Course is a form of e-learning platform. In recent years it has become very popular and is influencing modern educational thinking despite it emerging only 9 years ago [1]. There are two major types of MOOC: connectivist MOOC (cMOOC) and exponential MOOC (xMOOC). The key characteristic of cMOOC is a social networking integration between students that creates unique artefacts like images, videos and blog posts. A facilitator or a mentor can lead a CMOOC. Usually the mentor reviews and reflects on social activity, and other media output, produced by students and gives them advice or feedback. In contrast, a xMOOC is highly structured and consists of pre-determined sequenced periodical activities, which operates at a more selfpaced rate. Often XMOOCs contain units with text information and short videos. They use quizzes, as well as peer-reviewed assessments, to verify the learned material $[1,2]$. Numerous MOOCs can be found today on different MOOC platforms or providers such as Coursera, edX, Khan Academy, FutureLearn, Iversity, NovoEd, and so on.

Instructional design is a method for the development of learning models and activities. It is widely used by the course authors to design learning courses and educational materials but it can be used for the development of e-learning systems as well [3]. Examples of instructional design-based approaches for e-learning systems development are evidenced in systems such as IMS Learning Design (IMS LD) [4], MISA [5] and TELOS [6].

The Knowledge Field of Educational Environment with Competence Boundary Conditions (KFEEEC) is our generic instructional engineering method for the design of self-paced elearning systems. It is based on the TELOS [7] approach.

When we began to use KFEEC to design our MOOC platform, we faced some problems. Although KFEEC is quite flexible in providing a generic knowledge representation model to the different kinds of self-paced e-learning systems, it appears to be overcomplicated when being deployed in the MOOC platform. This paper describes the adoption of the KFEEC method to a MOOC platform design. It contains a brief description of KFEEC knowledge model, the steps of the adapting of KFEEC to the MOOC platform, the specification of the new knowledge model, and a discussion of our planned future work.

\section{KFEEC}

This section contains a brief description of the technologies and methods used in KFEEC and its associated knowledge model. A full and more detailed description can be found in our previous work [7]

\section{A. Instructional design}

As explained in the previous section, instructional design can be used to design e-learning systems. IMS LD is one of the first e-learning specifications to provide this ability [4]. It was created to substitute the old e-learning specifications intended to just transfer the classroom lessons to the computer. MISA expands IMS LD to the field of web-based e-learning systems. It is an instructional engineering method that distinguishes different knowledge types and abstract models [5]. TELOS is a 
base for KFEEC. TELOS is an evolution of the MISA approach. It uses an ontology to represent a course domain field, which is described using the Web Ontology Language (OWL) language [8].

\section{B. Ontology}

An ontology is defined as a specification of a conceptualization. Ontology is used as a knowledge representation of a domain or field to describe, share and reuse information that is not only in the form of data but in the form of a knowledge representation [9]. Design criteria for ontologies are clarity, coherence, extendibility, minimal encoding bias, and minimal ontological commitment [10].

\section{C. $O W L$}

OWL is a language for creating and describing ontologies for the Semantic Web. It is built on the top of the Resource Description Framework (RDF) and handled by description logic. The main concepts of OWL are classes, individuals, properties, and data values [8].

\section{KFEEC Knowledge model}

The KFEEC knowledge representation model is described using the OWL approach. This model is specified as an ontology that consists of defined and anonymous classes, object properties, data properties, and individuals. It uses logical constraints to describe the anonymous classes and to validate the consistency of the ontology [7].

KFEEC describes the domain field as a hierarchical structure of a parent and child classes. Its learning process is represented as a sequenced order of the learning objects. All learning objects have conceptual and functional information parts to represent the knowledge therein, as well as test and problem parts to verify that students have acquired this knowledge. Students can select their input competencies and desired output competencies. This allows student users to personalize their learning experience by devising an individual learning path. This path goes through all of the learning objects that connect the selected input and output competencies [7].

The KFEEC OWL ontology contains 13 classes, 14 object properties, 1 data property, and 9 logical constraints. These parts of the ontology, as well as the personalization mechanism, are mapped to the appropriate elements of the TELOS model. The KFEEC ontology is a task ontology, since it describes e-learning tasks for course management and learning, describes learning domains, and provides mechanisms for assessment [7]. The ontology class hierarchy is shown in Fig. 1.

During the design of our MOOC platform using KFEEC we encountered several problems, leading to the realization that its model should be simplified. This section describes our problems and the actions we took to solve them. Part of the problems came from the practical ontology usage experience and the other part of the problems is a technological limitation or overhead.

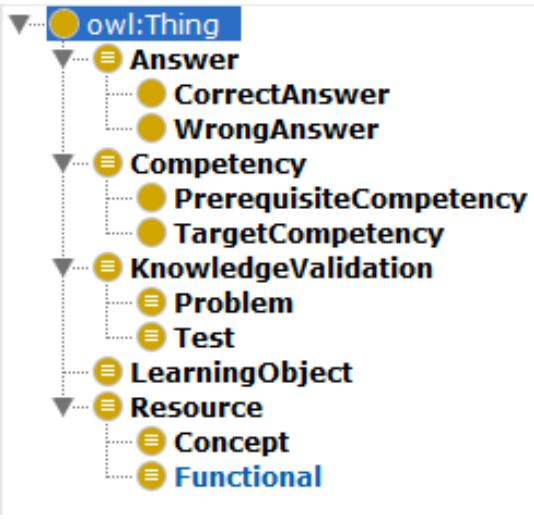

Fig. 1. Ontology class hierarchy.

\section{KFEEC ADAPTING}

\section{A. Problems}

- The ontology contains classes and anonymous classes that are not used for MOOC course design and learning. Such classes are created only for the generalization of the knowledge model.

- A number of object properties should be renamed or deleted if we want to remove unused regular and anonymous classes.

- Our ontology usage experience showed that the classes Answer, CorrectAnswer and WrongAnswer do not represent knowledge validation answers in a simple and clear logical way. The better representation of an answer is just one class with a simple IsCorrect Boolean data property.

- A full ontology consistency validation cannot be carried out for OWL ontologies because of the open world assumption OWL principle and limitations of current reasoners.

- Reasoners work relatively slowly for large ontologies. In our case it is better to completely avoid reasoning operations, for performance reasons, wherever possible.

- There is no practical advantage in the use of classes and an individual model. Every class individual should be manually supplied with every object and data property that the class has.

- Our scientific group is using the .NET Framework to develop the MOOC platform and other e-learning applications. Currently there is no usable tool or library for .NET to operate with OWL and to use reasoners. However, such libraries do exist for RDF.

- Considering the above issues, the best solution for our technical problems is to adapt the KFEEC knowledge model away from OWL to RDF with RDF Schema. 


\section{B. $R D F$}

It is important to define the RDF approach and to understand why its adoption is preferable over OWL in the case of this piece of work.

The key data structure of RDF is RDF graphs. Graphs are a set of triples: subject-predicate-object. Triples describe statements about resources. Usually subject and object are resources and predicate is a property. Graphs can be combined to a collection called a RDF dataset. RDF is a base for OWL. That is, OWL ontology is a correct RDF ontology [12].

A RDF Schema extends the RDF vocabulary with additional resources and introduces object-oriented mechanisms for RDF data [14].

\section{Solutions}

Below, we provide our solutions to the problems encountered and that were described in the previous subsection of the paper:

- All logical constraints were removed from the ontology: nine constraints for ontology consistency validation, as well as four constraints for the definition of the anonymous classes.

- Four regular classes were removed. All anonymous classes were removed automatically after our previous action.

- Class Answer was reorganized.

- Nine object properties were removed and three were renamed.

- We moved away from logical constraints and reasoners completely. This provided performance and simplification benefits.

- All manual technical operations, including the creation of properties for individuals, were moved to the application level.

- $\quad$ KFEEC knowledge model was heavily transformed and moved from OWL to RDF representation. We called this new model RDF MOOC Knowledge Model (RDF MOOC KM).

\section{RDF MOOC KM}

The RDF MOOC Knowledge Model is an adaptation and simplification of the KFEEC model for the usage in MOOC platform situations. It has a flat class and properties hierarchy. To provide a description of the model, the relationship between the different knowledge models and ontology languages described in Fig. 2.

\section{RDF classes (rdfs:Class):}

- LearningObject is core element of the model. It is the smallest unit of learning in a MOOC course. Learning objects depend upon each other: the knowledge obtained in learning obtained from the first object is a prerequisite for learning the second object and so on.
Learning object has connections with Concept, Functional, Test and Problem classes.

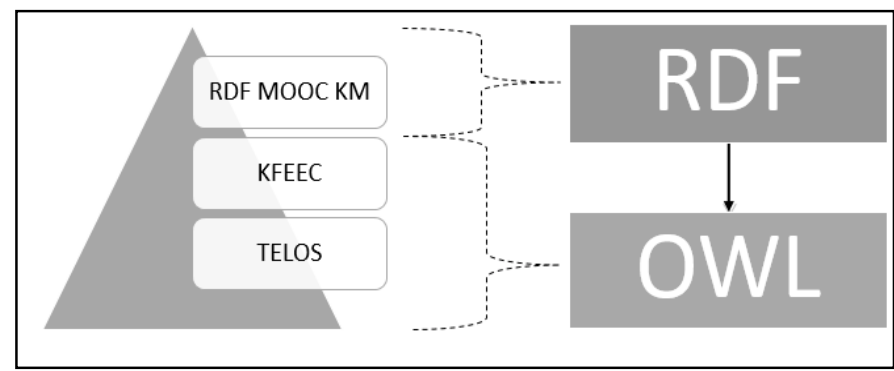

Fig. 2. The hierarchical representation of the described knowledge models and ontology representation languages.

- Concept represents conceptual knowledge about some LearningObject. It has a connection with LearningObject and Test classes.

- Functional represents functional knowledge about some LearningObject. It relates to the LearningObject and Problem classes.

- Test validates conceptual knowledge of a LearningObject. It has connections with the LearningObject and Concept classes.

- Problem validates functional knowledge of a LearningObject. It relates to LearningObject and the Functional classes.

- Answer contains a note of a correct or incorrect answer for Test or Problem and is connected with these classes.

- Competency indicates two things: the first is what a student should now start to learn from a LearningObject, and the second is what competency a student can acquire if they learn from aLearningObject.

RDF properties

- hasResource connects the LearningObject and Concept, Functional, Test and Problem classes.

- hasAssessment connects knowledge classes with appropriate validation classes: Concept and Test, Functional and Problem.

- hasAnswer relates assessment classes with answers: Test and Problem with Answer.

- isCorrect indicates whether some Answer correct or not. It connects Answer with literal of xsd:boolean data type.

- isFollowedBy represents connection between two LearningObject instances.

- isPrerequisiteFor and isTargetFor relate prerequisite and target competencies with appropriate learning objects. 
- hasContent connects Concept, Functional, Test and Problem classes with string literals. It represent content these resources.

This model provides the same abilities for MOOC platform development as KFEEC. It is still a task ontology but it is intended for use only in MOOC development.

\section{CONCLUSION}

We presented new knowledge representation model the design of MOOC platforms. It is based on KFEEC instructional engineering method and has more clear and simple structure that solves all stated model related problems. RDF MOOC KM described in RDF language. It is a task ontology with classes, properties and literals.

\section{FUTURE WORK}

We plan to continue our MOOC platform development. After RDF MOOC KM design the platform has all necessary theoretical background. MOOC platform will be created in a near future. Our next step will be content creation and testing of the platform.

\section{REFERENCES}

[1] F.M. Hollands and D. Tirthali, "MOOCs: Expectations and Reality. Full Report", Online Submission, ERIC, 2014.
[2] D. Levy, "Two Types of MOOCs: An Overview", Adult Education in Israel, vol. 13, 2014.

[3] G. Paquette, "Technology-Based Instructional Design - Evolution and Major Trends", Handbook of Research on Educational Communications and Technology, Springer, New York, 2014, pp. 661-671.

[4] R. Koper and B. Olivier, "Representing the learning design of units of learning", Journal of Educational Technology \& Society, JSTOR, vol. 7(3), 2004, pp. 97-111.

[5] G. Paquette, Instructional engineering in networked environments, John Wiley \& Sons; May 2004.

[6] G. Paquette, O. Mariño, D. Rogozan and M. Léonard, "Competencybased personalization for massive online learning", Smart Learning Environments vol. 2(1), 2015, pp. 1-19.

[7] B.G. Kiselev, V.A. Yakutenko and M.A. Yuriev,. "Knowledge acquisition and learning process description in context of e-learning", AIP Conference Proceedings, AIP Publishing, vol. 1797(1), p. 020007, 2017.

[8] J. Bao, "OWL 2 Web Ontology Language document overview", W3C Recommendation, vol. 201(2), 2012.

[9] T.R. Gruber, "A translation approach to portable ontology specifications", Knowledge acquisition, Academic Press, vol 5(2), 1993, pp. $199-220$.

[10] T.R. Gruber, "Toward principles for the design of ontologies used for knowledge sharing?", International journal of human-computer studies, Elsevier, vol. 43(5-6), 1995, pp. 907-928.

[11] T. Berners-Lee, J. Hendler and O. Lassila, "The semantic web", Scientific american, vol. 284(5), New York, 2001, pp. 28-37.

[12] R. Cyganiak, D. Wood and M. Lanthaler, "RDF 1.1 concepts and abstract syntax", W3C Recommendation, vol. 25, 2014, pp .1-8.

[13] D. Brickley, R.V. Guha and B. McBride, "RDF Schema 1.1", W3C recommendation, vol. 25, 2014, pp. 2004-2014. 